\title{
Clinical impact of PSMA PET/CT in primary prostate cancer compared to conventional nodal and distant staging: a retrospective single center study
}

Maarten L. Donswijk ${ }^{1 *}$ (D, Pim J. van Leeuwen², Erik Vegt ${ }^{1,3}$, Zing Cheung ${ }^{1}$, Stijn W. T. P. J. Heijmink4, Henk G. van der Poel ${ }^{2}$ and Marcel P. M. Stokkel ${ }^{1}$

\begin{abstract}
Background: To evaluate the impact of Gallium-68 $\left[{ }^{68} \mathrm{Ga}\right]$ labeled prostate specific membrane antigen (PSMA) positron emission tomography (PET)/X-ray computed tomography $(\mathrm{CT})$ compared with conventional imaging on staging and clinical management of men evaluated for primary prostate cancer (PCa).

Methods: Men with newly diagnosed biopsy-proven PCa who had been staged with a conventional staging protocol including bone scintigraphy (BS) and additionally underwent $\left[{ }^{68} \mathrm{Ga}\right] \mathrm{PSMA}$ PET/CT, were evaluated retrospectively. Imaging findings from BS, magnetic resonance imaging (MRI) and/or CT were categorized regarding locoregional nodal $(\mathrm{N})$ and distant metastasis $(\mathrm{M})$ status as negative, positive or equivocal before and after addition of the information of PET/CT. Also, the imaging-based level of confidence (LOC) in correct assessment of $\mathrm{N}$ and $\mathrm{M}$ status was scored. Impact of PET/CT on clinical management was evaluated by the percentage of treatment category changes after PET/CT as determined in the multidisciplinary tumour board.

Results: Sixty-four men with intermediate and high-risk PCa were evaluated. With additional information of PET/CT, N status was upstaged in 23\%, and downstaged in $9 \%$. M status was upstaged in $13 \%$, and downstaged in $23 \%$. A net increase in LoC of $20 \%$ was noted, mainly regarding M status.

Treatment category changed from palliative to curative in 9\%, and from curative to palliative in 3\%. An undecided treatment plan changed to curative in $14 \%$, as well as to palliative in another $9 \%$. In total, a $36 \%$ treatment category change was noted.

High negative predictive value of PET/CT for M status was indicated by 27 patients that underwent robot-assisted radical prostatectomy and reached postoperative biochemical disease-free status or had a likely other site of disease recurrence.

Conclusions: PSMA PET/CT can cause considerable changes in N and M staging, as well as in management compared to conventional staging. Findings of this study support the replacement of BS and CT by PSMA PET/CT in staging primary PCa.
\end{abstract}

Keywords: PSMA, Prostate, Staging, Management, Impact

\footnotetext{
*Correspondence: m.donswijk@nki.nl

'Department of Nuclear Medicine, The Netherlands Cancer Institute,

Plesmanlaan 121, Amsterdam, the Netherlands 1066, CX

Full list of author information is available at the end of the article
}

(c) The Author(s). 2020 Open Access This article is licensed under a Creative Commons Attribution 4.0 International License, which permits use, sharing, adaptation, distribution and reproduction in any medium or format, as long as you give appropriate credit to the original author(s) and the source, provide a link to the Creative Commons licence, and indicate if changes were made. The images or other third party material in this article are included in the article's Creative Commons licence, unless indicated otherwise in a credit line to the material. If material is not included in the article's Creative Commons licence and your intended use is not permitted by statutory regulation or exceeds the permitted use, you will need to obtain permission directly from the copyright holder. To view a copy of this licence, visit http://creativecommons.org/licenses/by/4.0/ The Creative Commons Public Domain Dedication waiver (http://creativecommons.org/publicdomain/zero/1.0/) applies to the data made available in this article, unless otherwise stated in a credit line to the data. 


\section{Background}

Management strategies for primary prostate cancer, whether with curative or palliative intent, have their own morbidities and costs. Recently estimated costs of prostate cancer therapies in Australia were US\$ $15 \mathrm{~K}-35 \mathrm{~K}$ per patient and US\$270.9 M in total, with an expected increase of $42 \%$ in 2025 [1]. Imaging has a pivotal role in staging of and selecting the appropriate management strategy in men with primary prostate cancer. Bone scintigraphy (BS) with Technetium-99 m [ $\left.{ }^{99 \mathrm{~m}} \mathrm{Tc}\right]$ labeled bisphosphonates has been the most widely used method for detecting bone metastases of $\mathrm{PCa}$, based on visualizing the increased osteoblastic activity of bone metastases [2]. Due to its moderate sensitivity and specificity, BS frequently results in equivocal findings regarding the presence of bone metastases [3-5]. Nonetheless, for primary staging of newly diagnosed $\mathrm{PCa}, \mathrm{BS}$ is still recommended for distant staging, combined with crosssectional abdominopelvic imaging for local and lymph node staging, in all high-risk patients [2].

After its clinical introduction in 2011, PET imaging with agents targeting the prostate-specific membrane antigen (PSMA), a transmembrane glycoprotein that is highly overexpressed on most PCa cells, has shown increasing adoption. Its value for staging recurrent $\mathrm{PCa}$ has already been well established [6].

More recently, PSMA PET/CT imaging was evaluated as a potential tool for staging primary prostate cancer in men prior to curative treatment [7]. Specificity rates of 84-100\% were reported by earlier studies [8-11] and in a recent systematic review [12]. However, the sensitivity of PSMA PET/CT for the detection of lymph node metastases is moderate (33-91\%), most likely due to the limitations in the spatial resolution to detect small $(<3 \mathrm{~mm})$ lymph node tumour deposits in primary as well as recurrent prostate cancer [13]. Its performance with regard to distant metastases and impact on clinical management compared to conventional staging is less investigated.

In our institute, a Gallium-68 $\left[{ }^{68} \mathrm{Ga}\right]$ labeled PSMA PET/CT was added to the conventional staging procedure in men with intermediate and high-risk PCa (predominant Gleason pattern 4 or higher, and/or cT3 or higher, and/or PSA blood level $\geq 20$ ) from June 2016 onwards. The aim of the present study is to evaluate the impact of additional PSMA PET/CT on staging and clinical management of men evaluated for primary $\mathrm{PCa}$ compared with a conventional staging protocol.

\section{Methods}

\section{Ethics approval}

This single-center was approved by the institutional review board of the Netherlands Cancer Institute and the need for written informed consent for usage of pseudonymized patient data was waived due to the retrospective nature of the study. Approval was registered under local number IRBd19063.

\section{Patients}

For this study, all patients with newly diagnosed biopsy proven PCa who had been staged between June 2016 and February 2018 with both $\left[{ }^{68} \mathrm{Ga}\right]$ PSMA PET/CT and BS were identified and selected from an institutional database, containing staging and treatment information. According to institutional protocol diagnostic procedures were performed before start of any treatment although androgen deprivation therapy (ADT) at time of imaging was allowed. Maximum time between conventional imaging and PSMA PET/CT was 90 days, which has been suggested as an acceptable interval for comparing staging modalities in PCa [4].

\section{Conventional staging}

Conventional staging included a clinical $\mathrm{T}$ stage assessment with digital rectal examination and PSA blood level measurement as well as conventional imaging including MRI, CT and BS. The MRI of the pelvis (multiparametric prostate protocol at a field strength of $1.5 \mathrm{~T}$ or $3 \mathrm{~T}$, without an endorectal coil) was evaluated by experienced radiologists according to PI-RADS v2. Conventional size criteria thresholds were used for lymph node evaluation [14]. BS consisted of planar images of the entire skeleton from anterior and posterior 3-4h after intravenous injection of approximately $555 \mathrm{MBq}$ $\left[{ }^{99 \mathrm{~m}} \mathrm{Tc}\right]$ labeled bisphosphonates, with additional detail views and / or SPECT/CT imaging of a body part if regarded necessary. All BS images were evaluated by experienced nuclear medicine physicians.

CT images were assessed for nodal and distant metastases. Generally, pelvic lymph nodes $>8 \mathrm{~mm}$ in maximum short axis diameter were regarded as positive. Either a contrast-enhanced abdominal CT (ceCT) was performed, or the lowdose CT (ldCT) as part of the PET imaging was used if no ceCT was performed. The ldCT was interpreted by two independent, experienced readers with clinical information but blinded from PET and other imaging results.

MRI, CT and BS findings were categorized according to the original clinical reports for regional nodal $(\mathrm{N})$ as well as distant metastases (M) staging: negative, positive or equivocal. For ldCT, the readers reached a consensus on $\mathrm{N}$ and $\mathrm{M}$ stage using the same categories. A composed conventional $\mathrm{N}$ and $\mathrm{M}$ stage was determined grouping the results of the MRI, CT and / or BS. Positive status on one of the conventional imaging modalities was regarded dominant over equivocal and negative results from the other modalities. Equivocal status was regarded dominant over negative results. The rationale is that modalities are complemental and scan ranges 
may not overlap, therefore a result that upstages the patient has to be disproven by additional imaging or procedures, and / or to be judged true or false when determining appropriate therapy.

\section{PSMA PET/CT staging}

Glu-NH-CO-NH-Lys-(Ahx)- $\left[{ }^{68}\right.$ Ga]-HBED-CC

$\left(\left[{ }^{68} \mathrm{Ga}\right]\right.$ PSMA-11) was used as tracer and produced on-site compliant to Good Manufacturing Practices regulations using a fully automated system (Scintomics GmbH, Germany). The tracer was administered to the patients as an intravenous bolus injection (100 MBq fixed dose). After an incubation period of $45 \mathrm{~min}$, PET imaging was performed from proximal femora to skull base on a Philips Gemini TF-II PET/CT scanner ( $3 \mathrm{~min} /$ bed for pelvis/abdomen and $2 \mathrm{~min} /$ bed position for the remainder), combined with a dose-modulated low dose CT (40 mAs, $2 \mathrm{~mm}$ reconstruction).

All PET/CT images were interpreted by nuclear medicine physicians with experience in prostate cancer PET imaging and reporting. Level of tracer uptake, location and morphological appearance of lymph nodes were considered to assess $\mathrm{N}$ status $[7,15,16]$. PET/CT imaging findings were categorized according to the original reports for regional nodal $(\mathrm{N})$ as well as distant metastases (M) staging: negative, positive or equivocal.

\section{Treatment policies}

Cases were discussed as part of standard clinical care in the tumour board (consisting of urologists, medical oncologists, radiation oncologists, radiologists and nuclear medicine physicians) with all diagnostic information available including PSMA PET/CT. A formal TNM staging and a preferred treatment were recorded in patients' chart. For patients with localized or regional lymph node metastases preferred treatment options with curative intent consisted of surgery or radiation (external or brachytherapy) whether or not combined with androgen deprivation. After therapy with curative intent, biochemical disease status was assessed with serum PSA measurements during follow up. Patients with high suspicion for distant metastases were considered for palliative treatment (ADT $+/$ - chemotherapy).

For purpose of the study, cases were discussed again in a smaller expert group (MD, PvL, HvdP) without information from PSMA PET/CT and a fictional TNM staging and preferred treatment was recorded in the study database.

\section{Impact of PSMA PET/CT on staging}

Per patient, the tumour $\mathrm{N}$ and $\mathrm{M}$ stage based on the composed conventional staging was compared to the tumour stage based on the additional information of the PSMA PET/CT. Differences in observed staging frequencies were tested for independence using a Chi-square test (IBM SPSS Statistics v25; Armonk, NY, USA). For both $\mathrm{N}$ and $\mathrm{M}$ staging, upstaging was defined as a change from negative to equivocal or positive, or from equivocal to positive. Downstaging was defined as a change from positive to equivocal or negative, or from equivocal to negative.

Furthermore, the level of confidence (LoC) in the correct assessment of the tumour stage based on the imaging findings was defined. This was done according to above mentioned $\mathrm{N}$ and $\mathrm{M}$ staging three-category systematics. For both $\mathrm{N}$ and $\mathrm{M}$ staging, increase in LoC was defined as a change from equivocal to positive or negative. Decrease in LoC was defined as a change from positive or negative to equivocal.

\section{Impact of PSMA PET/CT on clinical management}

Based on the recorded tumour stage with and without information from PSMA PET/CT, patients were divided into one of three categories of intended treatment: 'curative treatment', 'palliative treatment', or 'undecided'. 'Undecided' was assigned in case of equivocal $M$ stage based on the conventional staging. The impact of PSMA PET/ $\mathrm{CT}$ on clinical management was defined as a change of treatment category after additional PSMA PET/CT.

\section{Results}

\section{Patients}

Sixty-four men with a BS and a $\left[{ }^{68} \mathrm{Ga}\right] \mathrm{PSMA}$ PET/CT meeting the inclusion criteria were identified from the database (Fig. 1). Eight (13\%) had intermediate-risk PCa and $56(87 \%)$ had high-risk PCa. Four patients used the antiandrogenic oral drug bicalutamide and one patient used a gonadotrophin-releasing hormone analogue at time of PSMA PET/CT. Baseline patient characteristics are presented in Table 1. Performed imaging as part of the conventional staging protocol and median time between conventional imaging and PSMA PET/CT are presented in Table 2.

\section{Impact on staging}

Significant differences in $\mathrm{N}$ and $\mathrm{M}$ staging frequencies were found for CT and MRI, and for CT respectively compared to PSMA PET (Table 3). The linear weighted Kappa for the ldCT readers' agreement was 0.5 for $\mathrm{N}$ stage and 0.58 for $\mathrm{M}$ stage. Based on conventional staging, 41 patients (64\%) were staged as negative, ten $(16 \%)$ as positive, and thirteen $(20 \%)$ as equivocal for nodal metastases. With additional information of the PSMA PET/CT, 15 (23\%) were upstaged, six (9\%) were downstaged, and 43 (67\%) remained unchanged.

Based on conventional staging, 37 patients (58\%) were staged as negative, $12(19 \%)$ as positive, and $15(23 \%)$ as equivocal for distant metastases. With additional information of the PSMA PET/CT eight (13\%) were 


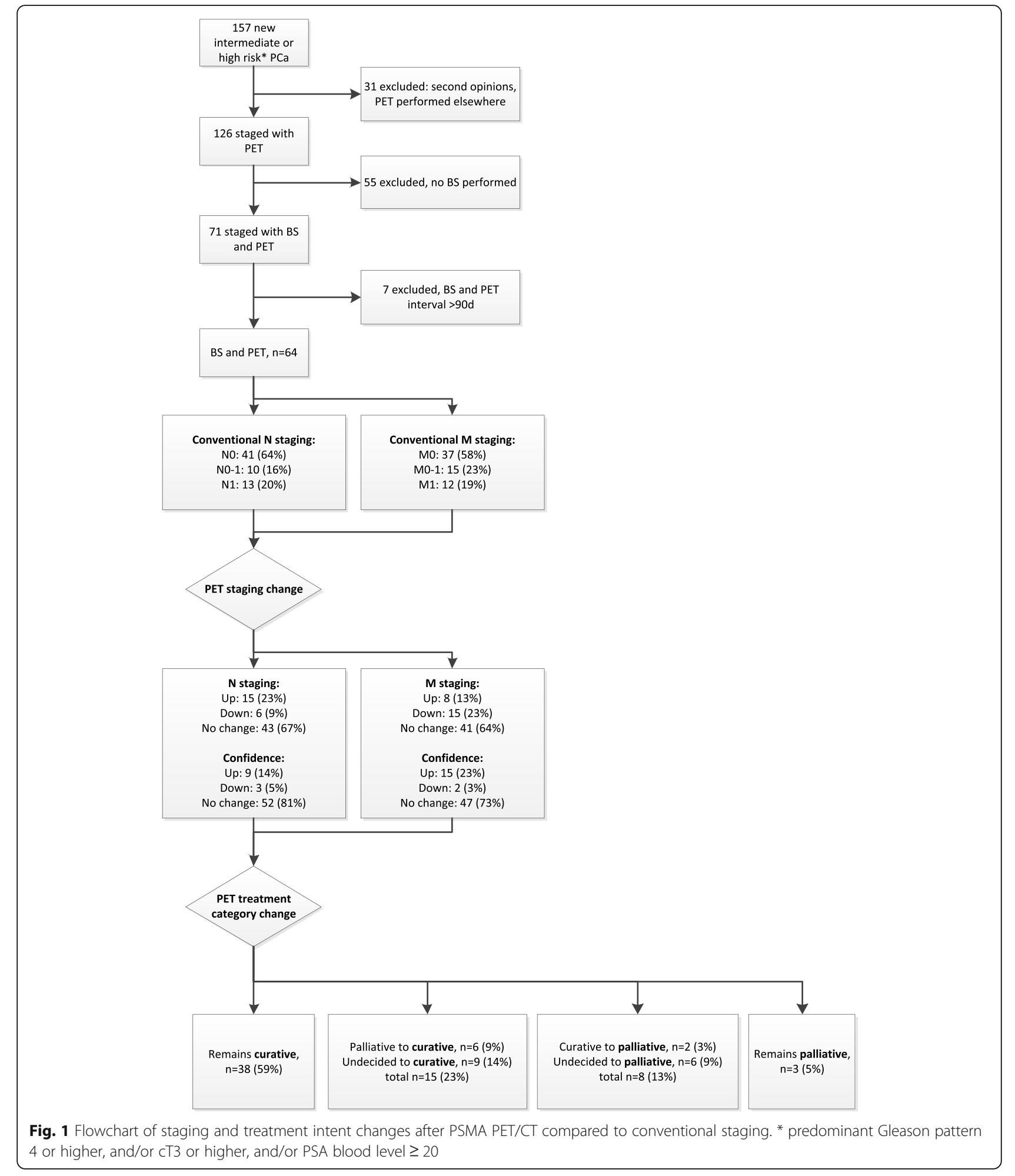

upstaged, 15 (23\%) were downstaged, and 41 (64\%) remained unchanged. A net increase in LoC of $20 \%$ was noted, mainly regarding $M$ status. Further frequencies and changes in staging and LoC are presented in Tables 3 and 4 .
Twenty-seven patients underwent RARP. Eighteen of these reached biochemical disease-free status (defined as serum PSA $<0.01$ after RARP without hormonal treatment), confirming absence of distant metastases as established with PET/CT staging. Notably, four of these 
Table 1 Baseline characteristics

\begin{tabular}{ll}
\hline Total number of patients & 64 \\
\hline Age in years, median (range) & $69(49-83)$ \\
PSA ng/ml, median (range) & $17(2,3-281)$ \\
Clinical tumour stage & \\
CT1C & $6(9 \%)$ \\
CT2a & $8(13 \%)$ \\
CT2b & $7(11 \%)$ \\
CT2C & $17(27 \%)$ \\
CT3a & $8(13 \%)$ \\
CT3b & $11(17 \%)$ \\
CT4a & $4(6 \%)$ \\
Gleason score & \\
6 & $3(5 \%)$ \\
$7 a^{a}$ & $9(14 \%)$ \\
$7 b^{b}$ & $8(13 \%)$ \\
8 & $29(45 \%)$ \\
$\geq 9$ & $15(23 \%)$ \\
Risk stratification & \\
Intermediate risk & \\
High-risk & $8(13 \%)$ \\
Y & $56(87 \%)$ \\
\hline No use at time of PSMA PET/CT & $59(92 \%)$ \\
\hline
\end{tabular}

Predominant Gleason score 3

${ }^{\mathrm{b} P}$ Predominant Gleason score 4

patients were staged positive and two equivocal with conventional staging.

Nine patients did not reach postoperative biochemical disease-free status. Eight of these were categorized as Mnegative, one as equivocal on PSMA PET. The most likely source of disease in these patients were remaining nodal metastases, because the majority of patients (8/9) had histologically proven nodal positive status after pelvic lymph node dissection (PLND) and follow-up PSMA $\mathrm{PET} / \mathrm{CT}$ in all patients within a year after surgery (median 7 months, range 3-11) showed nodal metastases as the only site of recurrence in five patients, no localization in three patients, and multiple localizations including lymph nodes in one patient.

\section{Impact on clinical management}

With additional information of the PSMA PET/CT, in 15 patients $(23 \%)$ the treatment category changed from palliative $(n=6)$ or undecided $(n=9)$ to curative. In eight patients $(13 \%)$ the treatment category changed from curative $(n=2)$ or undecided $(n=6)$ to palliative. In 41 patients $(64 \%)$ the treatment category based on conventional staging did not change.

The impact of PSMA PET/CT on treatment intent is presented in Table 5 and Fig. 1, and illustrated in Fig. 2.

\section{Discussion}

In this study, the clinical impact of additional diagnostic information from PSMA PET/CT was compared with conventional staging in patients with intermediate and high-risk prostate cancer. A considerable impact on both staging and clinical management was found. Compared to conventional primary staging of PCa, PSMA PET/CT changed $\mathrm{N}$ stage in $33 \%$ and $\mathrm{M}$ stage in $36 \%$, resulted in a net increase in the LoC of $9 \%$ for N status and of $20 \%$ for $\mathrm{M}$ status, and led to a treatment category change in 36\%.

A growing number of studies compared PSMA PET/ $\mathrm{CT}$ with conventional primary staging for primary PCa. Earlier studies found superior detection rates of PSMA $\mathrm{PET} / \mathrm{CT}$ for bone metastases compared to BS in primary PCa patients [3, 4, 17]. Furthermore, PSMA PET/CT induced management changes were reported by Roach

Table 2 Imaging characteristics

\begin{tabular}{|c|c|}
\hline Total number of patients & 64 \\
\hline \multicolumn{2}{|l|}{ Imaging performed } \\
\hline PSMA PET/CT & $64(100 \%)$ \\
\hline \multicolumn{2}{|l|}{ Bone scintigraphy } \\
\hline planar & $64(100 \%)$ \\
\hline $\mathrm{SPECT} / \mathrm{CT}$ & $30(47 \%)$ \\
\hline MRI prostate ${ }^{a}$ & $59(92 \%)$ \\
\hline \multicolumn{2}{|l|}{ CT abdomen } \\
\hline low dose & $54(84 \%)$ \\
\hline contrast enhanced ${ }^{\mathrm{b}}$ & $10(16 \%)$ \\
\hline Conventional imaging and PET/CT interval, median (range) & 49 days ( -12 to 87$)$ \\
\hline
\end{tabular}


Table 3 Comparison of conventional and PSMA PET/CT staging. $n=64$ patients

\begin{tabular}{|c|c|c|c|c|c|}
\hline \multicolumn{6}{|c|}{ A. Staging frequencies } \\
\hline \multicolumn{2}{|l|}{$\mathrm{N}$ stage } & $\mathrm{CT}$ & MRI & Conventional $^{a}$ & PSMA PET/CT \\
\hline \multicolumn{2}{|l|}{ negative } & $44(69 \%)$ & $43(67 \%)$ & $41(64 \%)$ & 38 (59\%) \\
\hline \multicolumn{2}{|l|}{ equivocal } & $9(14 \%)$ & $8(13 \%)$ & $10(16 \%)$ & $4(6 \%)$ \\
\hline \multicolumn{2}{|l|}{ positive } & $11(17 \%)$ & $8(13 \%)$ & $13(20 \%)$ & $22(34 \%)$ \\
\hline \multicolumn{2}{|l|}{ not available } & - & \multicolumn{3}{|l|}{$5(8 \%)^{b}$} \\
\hline M stage & Bone scintigraphy & $\mathrm{CT}$ & MRI & Conventional ${ }^{a}$ & PSMA PET/CT \\
\hline negative & 44 (69\%) & $54(84 \%)$ & $3(5 \%)$ & $37(58 \%)$ & $48(75 \%)$ \\
\hline \multirow[t]{3}{*}{ equivocal $^{a}$} & $9(14 \%)$ & $6(9 \%)$ & $5(8 \%)$ & $15(23 \%)$ & $3(5 \%)$ \\
\hline & • M1b: 9 (14\%) & - M1a: 2 (3\%) & \multicolumn{2}{|l|}{ - M1b: 9 (14\%) } & - M1b: 3 (5\%) \\
\hline & & - M1b: 4 (6\%) & & & \\
\hline \multirow[t]{4}{*}{ positive } & $11(17 \%)$ & $4(6 \%)$ & 0 & $12(19 \%)$ & $13(20 \%)$ \\
\hline & & & & & - M1a: 2 (3\%) \\
\hline & & & & & - M1b: 9 (14\%) \\
\hline & & & & & - M1c: 2 (3\%) \\
\hline \multicolumn{3}{|l|}{ not reported } & \multicolumn{3}{|l|}{51 (80\%) } \\
\hline not available ${ }^{b}$ & & & \multicolumn{3}{|l|}{$5(8 \%)$} \\
\hline
\end{tabular}

B. Contingency tables for observed staging frequencies

N stage

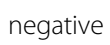

Conventional $^{\mathrm{a}}$

PSMA PET/CT

Totals

$X^{2}(2, N=64)=5.0 ; p=.0821$

negative

\section{CT}

PSMA PET/CT

Totals

44

38

82

$X^{2}(2, N=64)=6.0 ; p=.049076^{*}$

negative

MRI

43

PSMA PET/CT

Totals

38

81

$X^{2}(2, N=64)=8.0 ; p=.018451^{*}$

$M$ stage

$\begin{array}{ll} & \text { negative } \\ \text { Conventional }^{a} & 37 \\ \text { PSMA PET/CT } & 48 \\ \text { Totals } & 85 \\ X^{2}(2, N=64)=9.5 \cdot P=008811^{*}\end{array}$

negative

Bone scintigraphy

44

PSMA PET/CT

Totals

48

92

$X^{2}(2, N=64)=3.3 ; p=.188193$

$\begin{array}{lll}\text { equivocal } & \text { positive } & \text { Totals } \\ 10 & 13 & 64 \\ 4 & 22 & 64 \\ 14 & 35 & 128\end{array}$

equivocal positive Totals

$9 \quad 11$

64

64

128

equivocal

positive

Totals

8

4

12

22

59

64

123

equivocal positive Totals

$\begin{array}{lll}15 & 12 & 64\end{array}$

$\begin{array}{lll}3 & 13 & 64\end{array}$

$\begin{array}{lll}18 & 25 & 128\end{array}$

equivocal positive Totals

$9 \quad 11 \quad 64$

$3 \quad 13 \quad 64$

12

24 
Table 3 Comparison of conventional and PSMA PET/CT staging. $n=64$ patients (Continued)

\begin{tabular}{lllll}
\hline & negative & equivocal & positive & Totals \\
CT & 54 & 6 & 4 & 64 \\
PSMA PET/CT & 48 & 3 & 13 & 64 \\
Totals & 102 & 9 & 17 & 128 \\
$X^{2}(2, N=64)=6.1 ; p=.046943^{*}$ & & & \\
\hline
\end{tabular}

${ }^{a}$ composed conventional stage grouping results of bone scintigraphy, CT and MRI

${ }^{b}$ in 5 patients no MRI available due to evident distant metastases on PSMA PET/CT [3], due to severe claustrophobia [1] and performed elsewhere [1]

*denotes significance at alpha. 05 (Chi-square test of independence)

et al. in $21 \%$ of a larger cohort of primary PCa patients [18].

The recently published prospective multicentre proPSMA trial confirms the superior accuracy of PSMA $\mathrm{PET} / \mathrm{CT}$ to conventional imaging (CT and bone scintigraphy) in assessing pelvic nodal and distant metastatic disease (92\% vs 65\%) in high-risk primary PCa [19]. Furthermore, equivocal findings were less (7\% vs 23\%) and PSMA PET/CT conferred more management changes ( $28 \%$ vs $15 \%$ ). Also, when used as second-line imaging which is comparable to our study setting, PSMA PET/ CT resulted in significant upstaging of nodal status compared to conventional imaging ( $23,3 \%$ vs. $8,8 \%$ positive) and management changed occurred in $27 \%$.

The findings of our study are in line with abovementioned studies. In $33 \%$ of patients $\mathrm{N}$ stage was changed with information from PSMA PET/CT, mostly resulting in upstaging (23\%). Although correctness of these findings could not be formally assessed in our study, the high reported specificity of PSMA PET/CT for nodal metastases in primary staging of $\mathrm{PCa}$ suggests that upstaging would be correct in most cases, warranting considerable changes in patient management, such as increasing the extent of the PLND or the radiotherapy field [8-11, 20, 21].

PSMA PET/CT-induced treatment category changes in our study ranged from a conservative 13\% (curative to palliative, or vice versa) to an optimistic 36\% (any treatment category change). This is in line with abovementioned studies as well. Prostate cancer management is

Table 4 Impact of PSMA PET/CT staging and level of confidence on a per-patient level. $n=64$ patients

\begin{tabular}{lll}
\hline N stage & N Status & LOC $^{\mathrm{a}}$ \\
up & $15(23 \%)$ & $9(14 \%)$ \\
down & $6(9 \%)$ & $3(5 \%)$ \\
no change & $43(67 \%)$ & $52(81 \%)$ \\
M stage & $M$ Status & $L^{a}$ \\
up & $8(13 \%)$ & $15(23 \%)$ \\
down & $15(23 \%)$ & $2(3 \%)$ \\
no change & $41(64 \%)$ & $47(73 \%)$ \\
\hline a LoC: imaging-based level of confidence in correct assessment of N and \\
$M$
\end{tabular}

likely to be more and more patient-tailored and adjusted to staging information of imaging, such as tumour delineation in radiotherapy planning which may be signficantly influenced by PSMA PET/CT [21]. Findings that may not result in a considerable management change now, may do so in the future. The LoC in determining the correct $\mathrm{N}$ stage was considerably higher with PSMA $\mathrm{PET} / \mathrm{CT}$ compared to conventional staging, though equivocal results remained in up to $6 \%$. This is comparable with abovementioned studies as well. Further development of structured reporting criteria for negative and positive nodes may help to reduce the number of equivocal results and increase the LoC [15].

The major limitation of our study is the retrospective nature. Impact of PSMA PET/CT may be overestimated because patients may have been more likely to receive a PSMA PET/CT because of inconclusive findings on conventional imaging. Patients with overt metastases on BS probably are underrepresented because PSMA PET/CT may have been omitted due to lack of clinical consequences, and this may have overestimated staging and management impact of PSMA PET/CT as well. However, this reflects clinical practice as BS is cheaper and more readily available and may serve as an adequate first line $M$ staging tool in patients with highly elevated PSA levels. Furthermore, a true gold standard (histopathology) lacks in most cases, especially for distant metastases. The final diagnosis is substantially influenced by the findings of the PSMA PET/CT itself, leading to incorporation bias and overestimating the accuracy of PSMA $\mathrm{PET} / \mathrm{CT}$. The management changes described here however represent clinical practice. Although predefined rules were followed, the setting of the smaller unblinded expert group may have influenced the results of the fictional restaging and subsequent preferred treatment. Finally, the proportion of contrast-enhanced CTs in this study was low. Although literature showed poor sensitivity for $\mathrm{CT}$ in determining $\mathrm{N}$ stage and no established role in determining $M$ stage in primary $\mathrm{PCa}$, the comparison of PSMA PET/CT with conventional staging in our study may have been limited [22].

Use of ADT at time of PSMA PET imaging is allowed according to EANM procedure guideline on 68GaPSMA PET/CT imaging [23], however tracer uptake and 
Table 5 Treatment plan based on conventional vs PSMA PET/CT staging procedure. $n=64$ patients

\begin{tabular}{|c|c|c|c|c|}
\hline & Conventional staging & PSMA PET/CT staging & Impact of PSMA PET/CT & \\
\hline Curative & $40(63 \%)$ & $53(83 \%)$ & Remains curative & $38(59 \%$ \\
\hline Undecided & $15(23 \%)$ & 0 & Palliative to curative & $6(9 \%)$ \\
\hline \multirow[t]{4}{*}{ Palliative } & $9(14 \%)$ & $11(17 \%)$ & Undecided to curative & $9(14 \%)$ \\
\hline & & & Undecided to palliative & $6(9 \%)$ \\
\hline & & & Curative to palliative & $2(3 \%)$ \\
\hline & & & Remains palliative & $3(5 \%)$ \\
\hline
\end{tabular}

therefore PET staging results may be influenced by ADT [24]. As only five of 64 patients were undergoing hormonal therapy at time of PSMA PET, we do not think this has impacted our results.

The purpose of this study was not to assess the comparative accuracy of the conventional staging and the staging with PSMA PET/CT. Since histopathological confirmation of $\mathrm{N}$ status in the non-surgical treatment group lacked and confirmation of $\mathrm{M}$ status clinically was not feasible or did not have consequences, a true gold standard was not available. However, a high negative predictive value of PSMA PET/CT for $\mathrm{M}$ status is

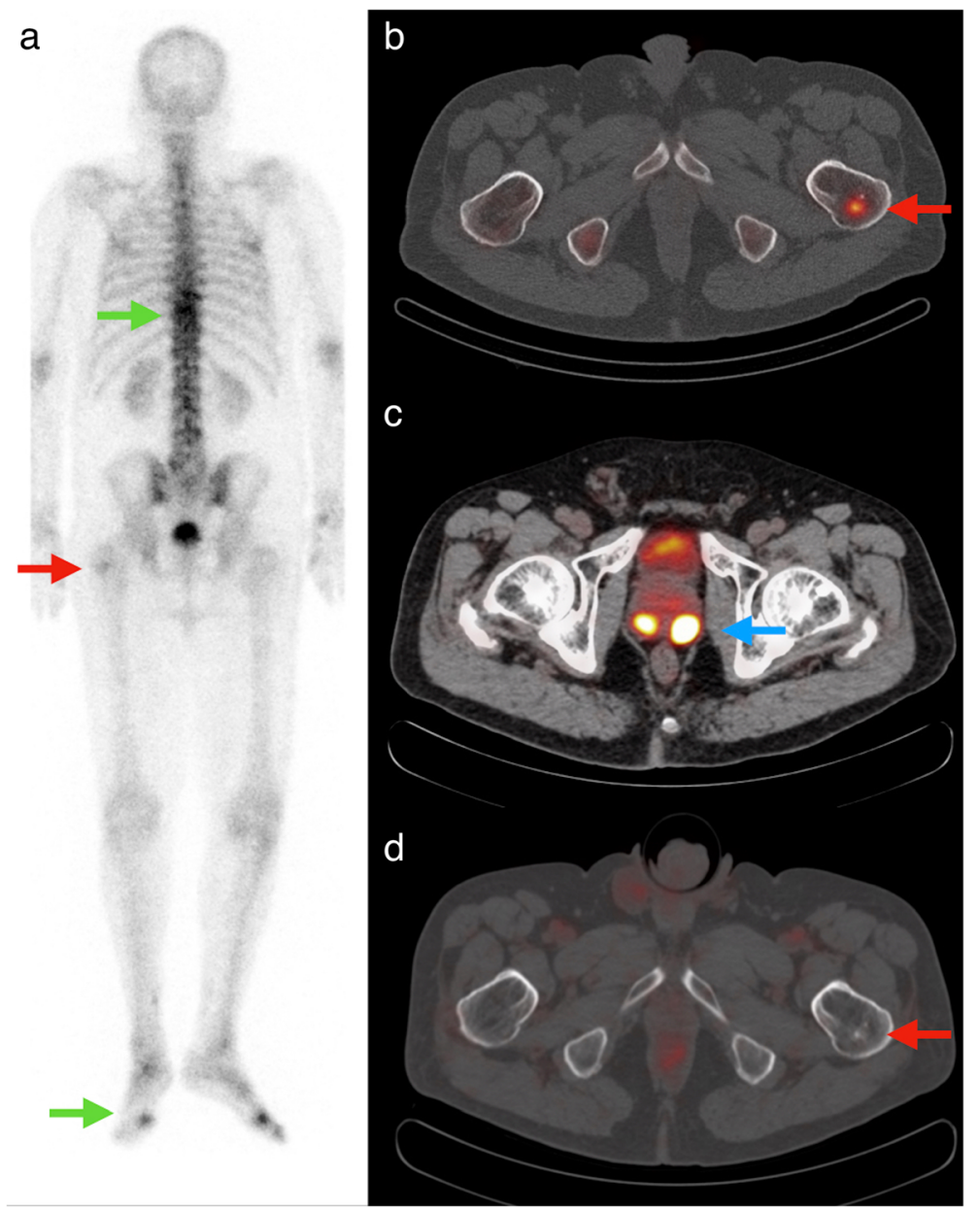

Fig. 2 Example of staging change of PSMA PET/CT compared with conventional imaging. A man presented with a clinical T2c Gleason 7b (predominant Gleason score 4) PCa with an iPSA of 16. Planar BS (a) showed a faint spot in the left proximal femur (red arrow) which was confirmed on SPECT/CT (b) as a sclerotic lesion with osteoblastic activity (red arrow). The lesion was interpreted as suspicious for bone metastasis. Other areas with increased osteoblastic activity (green arrows) were interpreted as degenerative. A $\left[{ }^{68} \mathrm{Ga}\right] \mathrm{PSMA}$ PET/CT (c) 36 days later shows a PSMA positive bilateral PCa (blue arrow), but no PSMA expression in the sclerotic lesion in the left proximal femur (d, red arrow). Based on PSMA PET/CT the lesion in the left proximal femur was regarded as not suspicious for bone metastasis; M stage changed from positive to negative and treatment intent changed from palliative to curative 
indicated by the 27 patients treated with RARP who reached biochemical disease-free status or were likely to have alternative sites of residual disease. Notably four of these patients were staged positive and two of them equivocal with conventional staging method. Thus, in addition to reported higher sensitivity of PSMA PET/CT for bone metastases compared to BS in primary staging of $\mathrm{PCa}$ [4], our data indicate a superior specificity of PSMA PET/CT compared to conventional staging.

\section{Conclusions}

Compared to conventional primary staging of $\mathrm{PCa}$, PSMA PET/CT changes $\mathrm{N}$ stage in 33\% and $\mathrm{M}$ stage in $36 \%$, leading to treatment category change in $36 \%$. BS was false-positive for distant metastases in $15-22 \%$ of cases. Findings of this study support the replacement of BS and CT by PSMA PET/CT in primary staging of $\mathrm{PCa}$, but prospective analyses are needed to confirm a possible beneficial effect on survival outcome.

\footnotetext{
Abbreviations

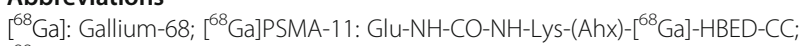
$\left[{ }^{99 \mathrm{~m}} \mathrm{Tc}\right]$ : Technetium-99 m; ADT: Androgen deprivation therapy; BS: Bone scintigraphy; ceCT: contrast enhanced X-ray computed tomography; CT: Xray computed tomography; ISUP: International Society of Urologic Pathologists; IdCT: lowdose X-ray computed tomography; LoC: Level of confidence; N: Regional nodal; M: Distant metastasis; M1a non-regional lymph node, M1b skeletal and M1c visceral metastasis; MRI: Magnetic resonance imaging; PET: Positron emission tomography; PCa: Prostate cancer; PLND: Pelvic lymph node dissection; (i) PSA: (initial) prostate specific antigen; PSMA: Prostate specific membrane antigen; RARP: Robot assisted radical prostatectomy
}

\section{Acknowledgments}

We thank Vincent van der Noort for giving statistical advice in presenting the data.

\section{Authors' contributions}

Conception of the work: MD, PvL, MS. Analysis: MD, PvL, ZC. Interpretation of data: MD, PvL, EV, ZC, SH, HvdP, MS. Draft and revision of the work: MD, PvL, EV, ZC, SH, HvdP, MS. All authors have carefully read and approve the revised manuscript.

\section{Funding}

None.

\section{Availability of data and materials}

The datasets generated and analysed during the current study are not publicly available as these contain individual person's data but are available from the corresponding author on reasonable request, after pseudonymization of the data and legal agreement.

\section{Ethics approval and consent to participate}

This single-center retrospective study was approved by the institutional review board of the Netherlands Cancer Institute and the need for written informed consent was waived. Approval was registered under local number IRBd19063.

\section{Consent for publication}

Not applicable.

\section{Competing interests}

None.

\section{Author details}

'Department of Nuclear Medicine, The Netherlands Cancer Institute, Plesmanlaan 121, Amsterdam, the Netherlands 1066, CX. ${ }^{2}$ Department of Urology, The Netherlands Cancer Institute, Amsterdam, the Netherlands. ${ }^{3}$ Department of Radiology and Nuclear Medicine, Erasmus MC, Rotterdam, Netherlands. ${ }^{4}$ Department of Radiology, The Netherlands Cancer Institute, Amsterdam, the Netherlands.

Received: 19 February 2020 Accepted: 17 July 2020

Published online: 05 August 2020

\section{References}

1. Gordon LG, Tuffaha HW, James R, Keller AT, Lowe A, Scuffham PA, et al. Estimating the healthcare costs of treating prostate cancer in Australia: a Markov modelling analysis. Urol Oncol. 2018;36(3):91 e7-e15.

2. Mottet N, van den Bergh RCN, Briers E, Comford P, De Santis S, Gillessen S, et al. Prostate cancer. Arnhem: EAU Guidelines Office; 2019.

3. Janssen JC, Meissner S, Woythal N, Prasad V, Brenner W, Diederichs G, et al. Comparison of hybrid (68) Ga-PSMA-PET/CT and (99m) TC-DPDSPECT/CT for the detection of bone metastases in prostate cancer patients: additional value of morphologic information from low dose CT. Eur Radiol. 2018;28(2):610-9.

4. Pyka T, Okamoto S, Dahlbender M, Tauber R, Retz M, Heck M, et al. Comparison of bone scintigraphy and (68)Ga-PSMA PET for skeletal staging in prostate cancer. Eur J Nucl Med Mol Imaging. 2016;43(12):2114-21.

5. Wondergem M, van der Zant FM, Knol RJJ, Burgers AMG, Bos SD, de Jong IJ, et al. (99m) Tc-HDP bone scintigraphy and (18) F-sodiumfluoride PET/CT in primary staging of patients with prostate cancer. World J Urol. 2018;36(1): 27-34.

6. Afshar-Oromieh A, Avtzi E, Giesel FL, Holland-Letz T, Linhart HG, Eder M, et al. The diagnostic value of PET/CT imaging with the (68) Ga-labelled PSMA ligand HBED-CC in the diagnosis of recurrent prostate cancer. Eur J Nucl Med Mol Imaging. 2015;42(2):197-209.

7. Corfield J, Perera M, Bolton D, Lawrentschuk N. (68) Ga-prostate specific membrane antigen (PSMA) positron emission tomography (PET) for primary staging of high-risk prostate cancer: a systematic review. World J Urol. 2018; 36(4):519-27.

8. Herlemann A, Wenter V, Kretschmer A, Thierfelder KM, Bartenstein P, Faber C, et al. (68) Ga-PSMA positron emission tomography/computed tomography provides accurate staging of lymph node regions prior to lymph node dissection in patients with prostate cancer. Eur Urol. 2016; 70(4):553-7.

9. Budaus L, Leyh-Bannurah SR, Salomon G, Michl U, Heinzer H, Huland H, et al. Initial experience of (68) Ga-PSMA PET/CT imaging in high-risk prostate cancer patients prior to radical prostatectomy. Eur Urol. 2016; 69(3):393-6.

10. Maurer T, Gschwend JE, Rauscher I, Souvatzoglou M, Haller B, Weirich G, et al. Diagnostic efficacy of (68) gallium-PSMA positron emission tomography compared to conventional imaging for lymph node staging of 130 consecutive patients with intermediate to high risk prostate cancer. J Urol. 2016;195(5):1436-43.

11. van Leeuwen PJ, Stricker P, Hruby G, Kneebone A, Ting F, Thompson B, et al. (68) Ga-PSMA has a high detection rate of prostate cancer recurrence outside the prostatic fossa in patients being considered for salvage radiation treatment. BJU Int. 2016;117(5):732-9.

12. Petersen $L$, Zacho HD. PSMA PET for primary lymph node staging of intermediate and high-risk prostate cancer: an expedited systematic review. Cancer Imaging. 2020;20(1):10.

13. Jilg CA, Drendel V, Rischke HC, Beck T, Vach W, Schaal K, et al. Diagnostic accuracy of Ga-68-HBED-CC-PSMA-ligand-PET/CT before salvage lymph node dissection for recurrent prostate cancer. Theranostics. 2017;7(6):1770-80.

14. Jager GJ, Barentsz JO, Oosterhof GO, Witjes JA, Ruijs SJ. Pelvic adenopathy in prostatic and urinary bladder carcinoma: MR imaging with a threedimensional TI-weighted magnetization-prepared-rapid gradient-echo sequence. AJR Am J Roentgenol. 1996;167(6):1503-7.

15. Rauscher I, Maurer T, Beer AJ, Graner FP, Haller B, Weirich G, et al. Value of 68Ga-PSMA HBED-CC PET for the assessment of lymph node metastases in prostate cancer patients with biochemical recurrence: comparison with histopathology after salvage lymphadenectomy. J Nucl Med. 2016;57(11):1713-9. 
16. Eiber M, Herrmann K, Calais J, Hadaschik B, Giesel FL, Hartenbach M, et al. Prostate cancer molecular imaging standardized evaluation (PROMISE): proposed miTNM classification for the interpretation of PSMA-ligand PET/ CT. J Nucl Med. 2018;59(3):469-78.

17. Lengana T, Lawal IO, Boshomane TG, Popoola GO, Mokoala KMG, Moshokoa E, et al. (68) Ga-PSMA PET/CT replacing bone scan in the initial staging of skeletal metastasis in prostate cancer: a fait accompli? Clin Genitourin Cancer. 2018;16(5):392-401.

18. Roach PJ, Francis R, Emmett L, Hsiao E, Kneebone A, Hruby G, et al. The impact of (68) Ga-PSMA PET/CT on management intent in prostate cancer: results of an Australian prospective multicenter study. J Nucl Med. 2018; 59(1):82-8.

19. Hofman MS, Lawrentschuk N, Francis RJ, Tang C, Vela I, Thomas P, et al. Prostate-specific membrane antigen PET-CT in patients with high-risk prostate cancer before curative-intent surgery or radiotherapy (proPSMA): a prospective, randomised, multicentre study. Lancet. 2020;395(10231):1208-16.

20. Schiller K, Sauter K, Dewes S, Eiber M, Maurer T, Gschwend J, et al. Patterns of failure after radical prostatectomy in prostate cancer - implications for radiation therapy planning after (68) Ga-PSMA-PET imaging. Eur J Nucl Med Mol Imaging. 2017;44(10):1656-62.

21. Calais J, Kishan AU, Cao M, Fendler WP, Eiber M, Herrmann K, et al. Potential impact of 68Ga-PSMA-11 PET/CT on the planning of definitive radiation therapy for prostate cancer. J Nucl Med. 2018;59(11):1714-21.

22. Gabriele D, Collura D, Oderda M, Stura I, Fiorito C, Porpiglia F, et al. Is there still a role for computed tomography and bone scintigraphy in prostate cancer staging? An analysis from the EUREKA-1 database. World J Urol. 2016;34(4):517-23.

23. Fendler WP, Eiber M, Beheshti M, Bomanji J, Ceci F, Cho S, et al. Ga-PSMA PET/CT: joint EANM and SNMMI procedure guideline for prostate cancer imaging: version 1.0. Eur J Nucl Med Mol Imaging. 2017;44(6):1014-24.

24. Emmett L, Yin C, Crumbaker M, Hruby G, Kneebone A, Epstein R, et al. Rapid modulation of PSMA expression by androgen deprivation: serial (68) GaPSMA-11 PET in men with hormone-sensitive and castrate-resistant prostate cancer commencing androgen blockade. J Nucl Med. 2019;60(7):950-4.

\section{Publisher's Note}

Springer Nature remains neutral with regard to jurisdictional claims in published maps and institutional affiliations.

Ready to submit your research? Choose BMC and benefit from:

- fast, convenient online submission

- thorough peer review by experienced researchers in your field

- rapid publication on acceptance

- support for research data, including large and complex data types

- gold Open Access which fosters wider collaboration and increased citations

- maximum visibility for your research: over $100 \mathrm{M}$ website views per year

At $\mathrm{BMC}$, research is always in progress.

Learn more biomedcentral.com/submissions 